\title{
Wire Rope Coupling Vibration Characteristics Analysis Based on Workbench
}

\author{
Yanan $\mathrm{Hu}^{1, a *}$, Huiping Feng ${ }^{2, b}$ and Huijuan Zhao ${ }^{3, c}$ \\ Mechanical and electrical technology department, Xijing University, Shaanxi Xi'an, China \\ 2664961273@qq.com
}

Keywords: Vibration characteristics; Flexible coupling; Frequency; Steel wire rope

\begin{abstract}
In this paper, the main vibration frequency is obtained by the finite element analysis of the shaft joint, which can avoid the resonance of the wire rope coupling system in the actual work. The finite element model of slow-speed of revolution coupling is established. The model is used to simulate the actual production model of the coupling system. The modal analysis of the natural frequency and the main vibration mode of the two kinds of motion limit states are carried out. The workbench analysis results showed that the natural frequency of the low speed and the working state of the wire rope coupling changes little, and the vibration mode is consistent. The main vibration modes correspond to a certain frequency range. The results of this paper provide the reference data for the control of the sugarcane layer in the actual production. The safe operation of sugarcane juice extractor is guaranteed.
\end{abstract}

\section{Introduction}

The flexible coupling of steel wire rope is an important connecting device of large rotary equipment. The vibration characteristics of the coupling directly affect the reliability of the overall operation, life, economic indicators. Resonance is one of the important reason of the failure of wire rope fatigue and structural breaks[1]. The reason is that the frequency of the external excitation is close to the natural frequency of the rope coupling.

\section{Flexible Wire Rope Coupling}

Structural Analysis. The concrete structure of the flexible coupling is shown in Fig. 1. The flexible coupling of wire rope consists of square coupling(1), active end of coupling(2), the floating supporting plate(3,7), driven end of coupling(4), wire rope rigging(5), ring sling(6)and laminate(8)[2]. The four steel wire ropes are respectively connected with the left and the right coupling. The floating connecting block is arranged between the two couplings, and is pulled by a steel rope rigging. Both ends of the square coupling are respectively connected with the press and the speed reducer. The floating connecting block in the middle of the two coupling is used for supporting, and is respectively connected with the four steel wire ropes of the coupling.
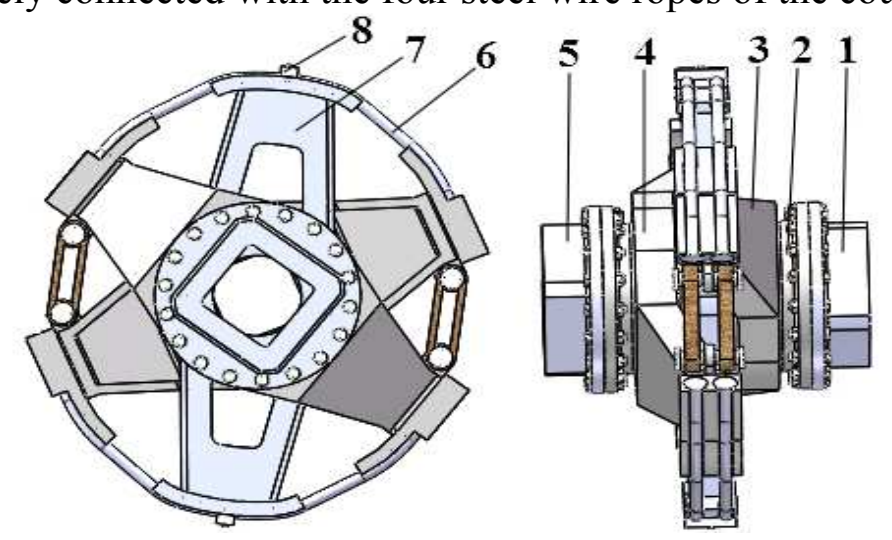

Figure 1. Structure of wire rope coupling 
Principle Analysis. When the equipment is running, the active end and the driven end of the coupling are respectively connected with the output end of the transmission system and the input end of the heavy equipment. The four rings are respectively connected with the left and the right coupling, which is used to transfer the smaller torque when the equipment is reversed. When the top roller of the juicer rises or falls, the square coupling will rise or fall together. At the same time, one side of the coupling of the two wire rope rigging will be tight, while the other side of the two wire rope rigging will relax. The floating support plate moves to the two steel wire ropes which are relaxed, so as to ensure the tension of the two steel rope rigging on both sides of the four steel rope. The coupling of the transmission system to maintain the original state will not rise or fall [3].

Motion State Analysis. In the actual production process, the actual thickness of the layer will not exceed $50 \mathrm{~mm}$. The coupling is based on the actual production of structural size design. In the process of movement, the steel wire rope can be separated from the coupling track and the floating supporting plate track to compensate the error of the shafting. In addition, the degree of detachment of the wire rope is based on the rotation position of the driven end coupling [4]. As shown in Fig.2,The radial fluctuation caused by the stacking of the sugarcane layer is always in the vertical direction, so the floating direction can be decomposed into the compensation amount perpendicular to the middle surface of the driven end joint and the amount of compensation parallel to the middle plane. Fig.3 is the state diagram of the radial error compensation along the center of the driven end of the wire rope. Because the steel rope, the coupling and the annular chain form a completely mutually controlled integral. Therefore, when the radial error of the vertical driven end joint is compensated, the steel wire is not separated from the coupling and the floating supporting plate. Based on the literature [5] and after deformation calculation shows: when the radial component of the middle surface of the driven end is full radial floating, the degree of the steel cable is separated from the floating support plate to reach the maximum. At this time the whole coupling system stiffness is the smallest.

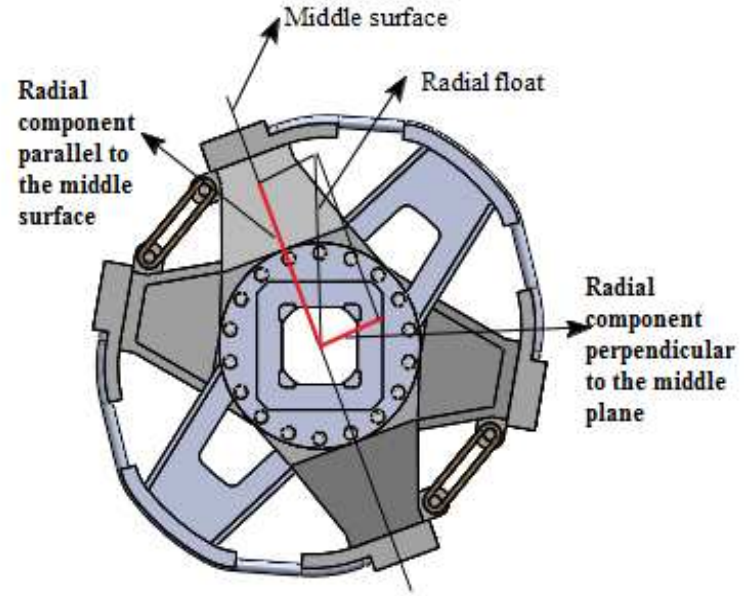

Figure2. Schematic of radial vibration

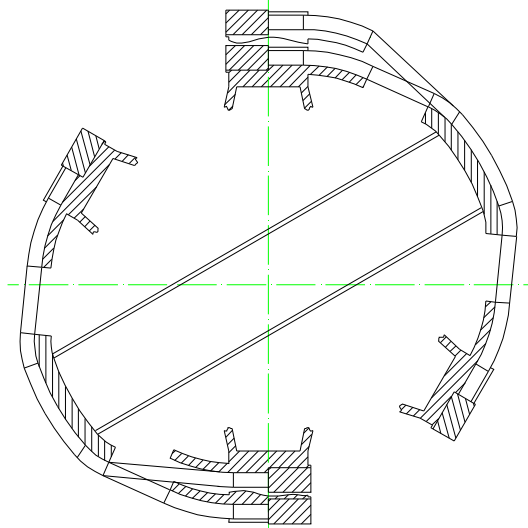

Figure3. Schematic diagram of vibration

\section{Modal Analysis}

Model Simplification. It can improve the accuracy of the model by simplifying the model and reducing the degree of meshing.

Finite Element Processing. Taking into account the structural characteristics of the system is more complex, this paper takes the design dimensions as the standard geometric parameters. The establishment of three-dimensional model of wire rope coupling in Solid works [6]. The interface between Solidworks and Workbench is used to import the model into Workbench interface for finite element analysis. As shown in Table 1, the material parameters of each part of the assembly. 
Table. 1 material properties of each part

\begin{tabular}{ccccc}
\hline Part Number & Material & Density $\left(\mathrm{kg} / \mathrm{m}^{3}\right)$ & Poisson ratio & Elastic modulus (GPa) \\
\hline 1 & ZG270-500 & 7800 & 0.3 & 202 \\
5 & QT & 7300 & 0.3 & 173 \\
6 & 40Cr & 7850 & 0.28 & 206 \\
7 & 70\# Steel & 7810 & 0.304 & 206 \\
8 & Q235 & 7858 & 0.3 & 200 \\
\hline
\end{tabular}

The wire rope and the coupling, floating plate, pressure plate contact mode is set to No separation, the rest are set to Bonded. In addition, the partition mesh is a tetrahedral element type. According to the actual load situation, the active and the driven end of each add a Romote Displacement constraint, the active end of the axial direction of rotation is set to Free, the driven end of the radial movement is set to Free[7].

Results Extraction and Analysis. The coupling system under steady state and maximum amplitude is analyzed and the results are obtained. It can be seen from the results that the 1 order natural frequencies of the two states are close to 0 , because the axial rotation of the coupling system belongs to the rigid body motion[8-9]. According to the classical vibration theory, the low order natural frequency of the structure has a great influence on the dynamic response of the system. In this paper, the natural frequencies and mode shapes of the first 3 modes of the wire rope flexible coupling system under steady state and maximum amplitude are extracted. As shown in Fig. 4 is second order and three order vibration mode diagram in steady state. As shown in Fig. 5 is second order and three order vibration mode diagram under the maximum amplitude.

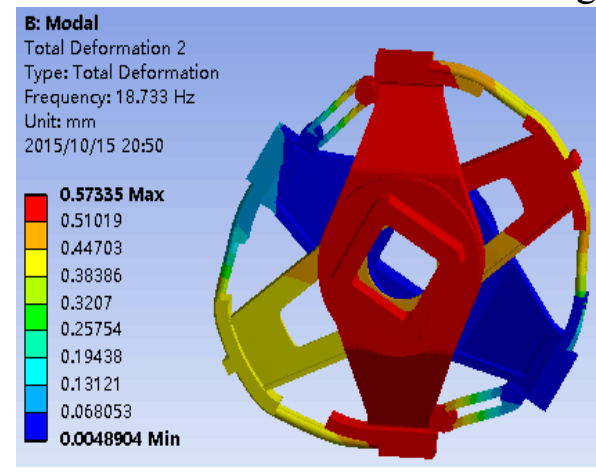

(a) Second order vibration mode diagram Figure. 4 Second order and three order vibration mode diagram in steady state

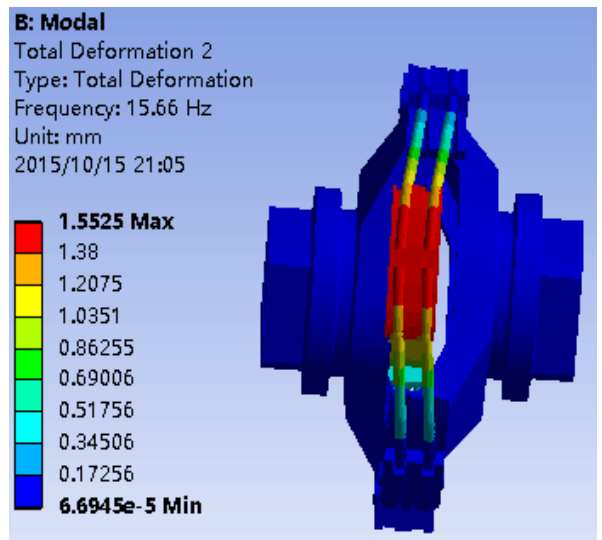

(a) Second order vibration mode diagram

Figure. 5 Second order and three order vibration mode diagram under the maximum amplitude

As shown in Table 2, the frequency and the main vibration mode of wire rope coupling are obtained by finite element analysis. 
Table. 2 Natural frequencies and modes of vibration

\begin{tabular}{|c|c|c|c|}
\hline Order & Frequency & Maximum amplitude & Principal mode \\
\hline 1 & 15.66 & 0.570 & Radial vibration of active end \\
\hline 2 & 18.71 & 1.553 & Axial vibration of floating plate \\
\hline 3 & 24.72 & 1.986 & $\begin{array}{l}\text { Swinging along the axial direction of the } \\
\text { floating plate }\end{array}$ \\
\hline 4 & 39.24 & 1.966 & $\begin{array}{c}\text { Twisting along the centerline of the floating } \\
\text { plate }\end{array}$ \\
\hline 5 & 41.75 & 1.876 & $\begin{array}{l}\text { Vibration along the center line of the vertical } \\
\text { floating plate }\end{array}$ \\
\hline 6 & 58.88 & 1.793 & Rotation along the axis of the floating plate \\
\hline
\end{tabular}

\section{Conclusions}

By solving the natural frequency under the condition of steady state and maximum vibration, the natural frequency range of the large steel wire rope coupling system at low speed rotation is analyzed. Comparative analysis of actual condition and the modal results can accurately determine whether the wire rope coupling system resonance will occur in practical work. The measures to avoid resonance is to make the frequency range of the external excitation as far as possible to stagger the natural frequency range of the coupling system. The result of modal analysis is the basis for the reasonable design of the coupling system. In addition, the results of modal analysis can be used as the basis for fault diagnosis and other dynamic analysis of the coupling system.

\section{References}

[1] FU Zhi-fang, HUA Hong-xing. Modal analysis theory and Application [M]. Shanghai, Profile of Shanghai Jiao Tong University Press, 2000: 1-44.

[2] YANG Wei, MA Xing-guo, YOU Xiao-mei. Modal analysis of gear assembly based on ANSYS [J]. Journal of Shenyang Li Gong University, 2008, 27(4): 71-74.

[3] LIU Cong-cong, FAN Jun. Dynamic analysis and Simulation of wire rope of linear motor pumping unit based on Simulink [J]. Mechanical design and manufacturing, 2013, (1): 188-190.

[4] LI Bing, GAO Dong-qiang, LI Tian-yi. Optimization and analysis of structural parameters of flexible wire rope coupling [J]. Coal mine machinery, 2015, 36(7): 198-200.

[5] LI Qiang, YAN Yue, YAN Hong-bo. Study on stress field and fatigue life of steel wire rope [J]. Mechanical design and manufacturing, 2014, (10): 199-122.

[6] LI Jie, XIANG Chang-le. Nonlinear modal analysis of gear in high speed rotating state [J]. Modern manufacturing engineering, 2007, (7): 77-79.

[7] GAO Dong-qiang, LI Bing, WANG Wei. Influence of vibration amplitude on fatigue life of wire rope coupling [J]. Coal mine machinery, 2015, 36(7): 148-151.

[8] YUAN Fang, HU Bin-liang, ZHOU Zhi-jin. Research status and Prospect of defect detection method for in-service steel wire rope [J]. Mechanical design and manufacturing, 2010, (2): 260-262.

[9] LI Qiang, YAN Yue, YAN Hong-bo. Modal analysis of logarithmic spiral bevel gear assembly based on ANSYS [J]. Mechanical design and manufacturing, 2013, (5): 194-196. 